\title{
Congenital right subclavian artery-superior vena cava fistula recognized by transthoracic echocardiography
}

\author{
Manwei $\mathrm{Liu}^{1,2, *}$, Yali Yang ${ }^{1,2, *}$, Wenqian $\mathrm{Wu}^{1,2}$, \\ Li Zhang ${ }^{1,2}$, Yuman $\mathrm{Li}^{1,2}$, Mingxing Xie ${ }^{1,2}$ \\ ${ }^{1}$ Department of Ultrasound, Union Hospital, Tongji Medical College, \\ Huazhong University of Science and Technology, Wuhan, China \\ ${ }^{2}$ Hubei Province Key Laboratory of Molecular Imaging, Wuhan, China
}

A 16-year-old girl presenting with dyspnea and chest pain was admitted to the documented institution. On physical examination, a grade $3 / 6$ continuous murmur was audible on the right sternal border at the $2^{\text {nd }}$ and $3^{\text {rd }}$ intercostal spaces. Standard transthoracic echocardiography (TTE) images via conventional acoustic windows revealed mildly dilated right atrium and right ventricle, mild tricuspid regurgitation and mild pulmonary hypertension. This could not explain the murmur. However, imaging in unconventional right parasternal windows, which was close to the site of the murmur, revealed a dilated right subclavian artery (RSA) with a 7-mm fistula to the superior vena cava (SVC) (Fig. 1A-C; Suppl. Video 1). Continuous-wave Doppler showed flow signals measuring $3.5 \mathrm{~m} / \mathrm{s}$ continuously moving from the RSA into the SVC throughout the cardiac cycle, consistent with the fistula (Fig. 1D). Subsequent computed tomographic angiography confirmed a congenital arteriovenous fistula (AVF) between the RSA and the SVC (Fig. 1E, F). The patient was referred for transcatheter occlusion of the fistula. The arterial angiography showed contrast material leaking from the RSA into the SVC (Fig. 1G). The fistula was successfully closed using a 10/12 mm Amplatzer Ductal Occluder (Fig. 1H). The postprocedure TTE revealed no residual shunt (Fig. 1I). The patient dramatically improved clinical symptoms and made an uneventful recovery after occlusion.

Congenital AVFs are very rare and are usually diagnosed by angiography. Presented herein, an adolescent patient with a congenital RSA-SVC fistula was first recognized by TTE. The present case highlights the need for clinical suspicion of congenital AVFs in unusual locations when evaluating a patient with unexplained cardiac murmur.

Conflict of interest: None declared

Address for correspondence: Mingxing Xie, MD, PhD; Yuman Li, MD, PhD, 1277 Jiefang Avenue, Wuhan, China, tel: 86-27-85726430, fax: 86-27-85726386, e-mail: xiemx@hust.edu.cn; liym@hust.edu.cn

Received: 12.08.2019 Accepted: 3.11.2019

*Both authors contributed equally to this manuscript. 


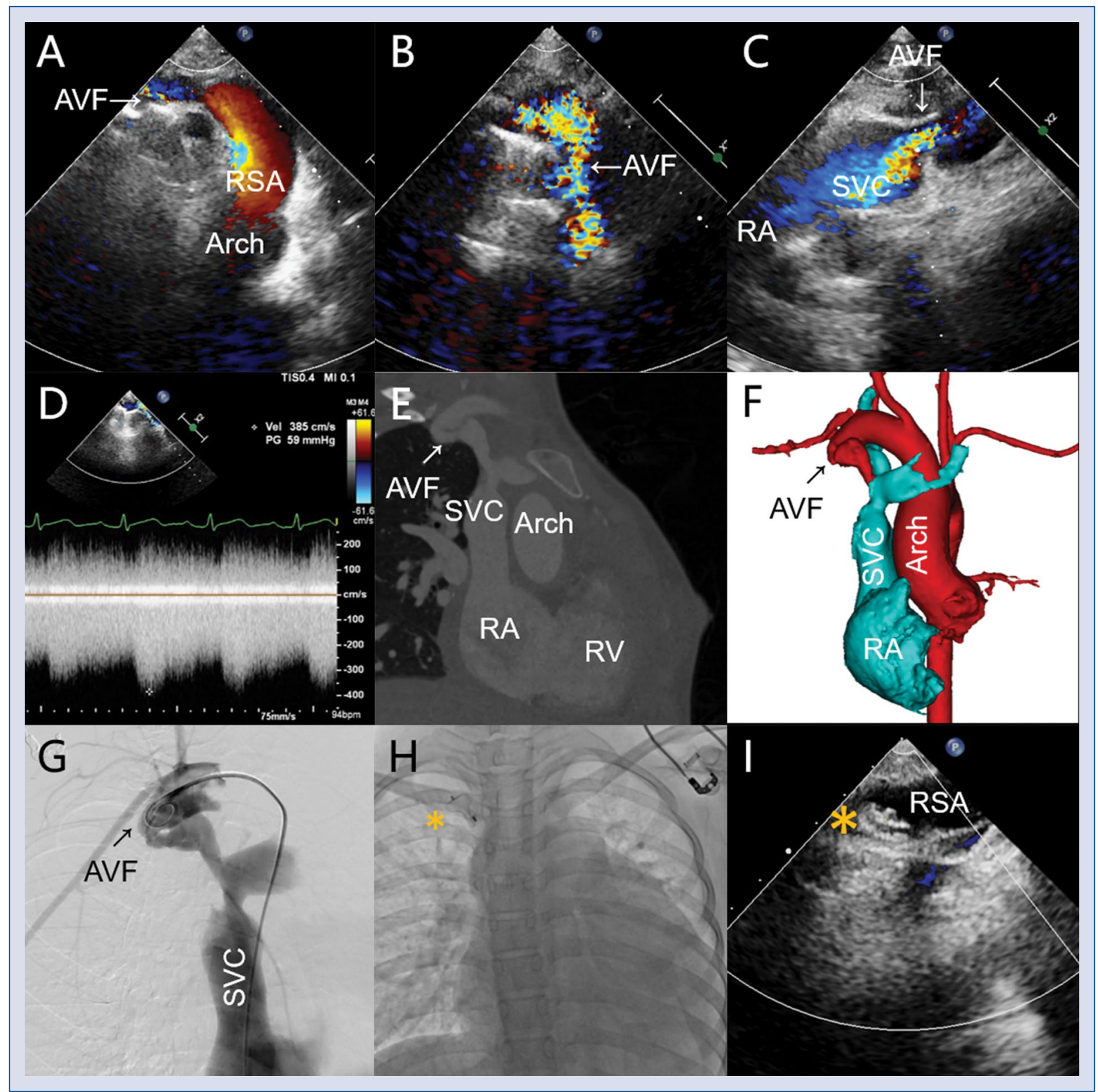

Figure 1. A-C. Transthoracic echocardiography shows the arteriovenous fistula (AVF, arrow) between the right subclavian artery (RSA) and the superior vena cava (SVC); D. Color Doppler guided continuous-wave Doppler interrogation of the fistula shows high velocity flow signals moving from the RSA into the SVC throughout the cardiac cycle; E, F. Computed tomographic angiography and three-dimensional reconstruction shows AVF (arrow); G. Arterial angiography indicates contrast material leaking into the SVC through the AVF (arrow); $\mathbf{H}$. The fistula is successfully closed using a 10/12 mm Amplatzer Ductal Occluder (asterisk); I. Transthoracic echocardiography shows no residual shunt; RA — right atrium; RV — right ventricle. 